\title{
Effects of Periodontal Treatment on Glycated Hemoglobin A Levels in Patients with Type 2 Diabetes: A Meta-Analysis of Randomized Clinical Trials
}

\author{
So-Hyun Son and Eun-Sun Lee ${ }^{1, \dagger}$ \\ Department of Dental Hygiene, Shingu College, Seongnam 13174, \\ ${ }^{1}$ Department of Dental Hygiene, Kyungbok University, Pocheon 11138, Korea
}

\begin{abstract}
This systematic review aimed to investigate the effects of periodontal treatment on glycated hemoglobin A (HbA1c) levels in patients with type 2 diabetes who develop periodontal disease. The search of the MEDLINE, Embase, CINAHL, and Cochrane Library databases was completed on April 8, 2018. The study design was based on randomized clinical trials. Scaling and root planing was performed for the test group, whereas no periodontal treatment or simple oral training was performed for the control group. The main outcome variable was the change in $\mathrm{HbA} 1 \mathrm{c}$ levels. We used the Review Manager statistical analysis software for the quantitative analysis of selected documents. Meta-analysis was performed using the inverse variance estimation method of the fixed-effect model to estimate the effects of periodontal treatment on $\mathrm{HbA} 1 \mathrm{c}$ levels in patients with type 2 diabetes. A total of 1,011 documents were searched using search strategies, and 10 documents were included in the meta-analysis. The meta-analysis of the selected literature showed that periodontal treatment significantly reduced the HbA1c levels in patients with type 2 diabetes who develop periodontal disease (mean difference, $-0.34 ; 95 \%$ confidence interval, -0.43 to $-0.26 ; p<0.001$ ). This study aimed to investigate the effects of periodontal treatment on $\mathrm{HbA1c}$ levels, which can be used as a basis for the increasing management of diabetic complications. To improve the quality of life and reduce the burden of medical expenses for patients with diabetes, periodontal disease management through nonsurgical periodontal treatment, such as scaling and root planing, is necessary.
\end{abstract}

Key Words: Dental scaling, Glycated hemoglobin A, Meta-analysis, Periodontal diseases, Type 2 diabetes mellitus

\section{Introduction}

Diabetes is one of the most common chronic diseases worldwide ${ }^{1)}$. As the level of physical activity decreases and the incidence of obesity increases due to lifestyle changes, the importance of diabetes consistently increases ${ }^{1}$. The prevalence of diabetes worldwide is $6.4 \%$, and it is expected to increase up to $7.7 \%$ by 2030 , resulting in a health burden at the international level ${ }^{1}$. The prevalence of diabetes in Korea is $8.23 \%$, and it is higher than that in other countries ${ }^{2}$. Korea has become an aging society at a rapid rate and is awaiting its transition into a super-aged society; hence, the population of patients with diabetes is expected to increase rapidly ${ }^{3)}$.
Type 2 diabetes, which is the most common type of diabetes, is characterized by complications of hyperglycemia and hyperlipidemia ${ }^{4)}$. Periodontal diseases are the sixth most common complication of diabetes and can affect other diabetes complications such as retinopathy and coronary artery diseases ${ }^{5)}$. Periodontal diseases and diabetes interact with one another ${ }^{6,7)}$. In patients with periodontal diseases, the levels of systemic inflammatory mediators, such as interleukin-6, tumor necrosis factor (TNF)- $\alpha$, and C-reactive proteins (CRP), which reduce insulin resistance, are elevated, which can decrease blood glucose control $^{6,7)}$.

Periodontal diseases are infectious diseases caused by dental plaques ${ }^{8)}$. In a long-term clinical study, scaling and root planing (SRP) was found to have therapeutic effects ${ }^{8)}$. 
The goal of nonsurgical periodontal treatment is to convert bacterial flora that cause periodontal diseases into healthy bacterial flora, to eliminate inflammatory periodontal pockets, to convert deep periodontal pockets into healthy gingival sulcus, and make the root surface appropriate for attachment to healthy epithelium and connective tissue ${ }^{8)}$.

Reviewing previous findings on the association between diabetes and periodontal diseases, diabetes education, diabetes treatment, and diabetes-related characteristics, such as glycated hemoglobin $\mathrm{A}(\mathrm{HbA} 1 \mathrm{c})$, were associated with the prevalence of periodontal diseases, as reported in a study conducted by Jung et al. ${ }^{9}$. In Won and Ha's study ${ }^{10)}$, the risk of impaired fasting glucose was twice as high among individuals with a community periodontal index of 2 or below than among those with an index of 3 or higher. Kim and Jang's study ${ }^{11)}$ on the association between metabolic syndrome and periodontal diseases in Korean adults showed that hypertension, diabetes, and dyslipidemia were associated with periodontal diseases.

Several interventional studies have hypothesized that periodontal treatment can improve metabolic control in patients with diabetes.

Iwamoto et al. ${ }^{12)}$ administered minocycline to patients with type 2 diabetes for 1 month. Their results showed that the number of microorganisms in periodontal pockets, TNF- $\alpha$, glycosylated hemoglobin, and fasting insulin were significantly reduced ${ }^{12)}$. However, in Jones et al.'s study ${ }^{13)}$, no significant difference was observed between individuals who underwent periodontal treatment and the control group, 4 months after the treatment. As can be seen, study results have been inconsistent.

In a systematic review by Janket et al. ${ }^{14)}$ that analyzed interventional studies on the effects of periodontal treatment on blood glucose control, periodontal treatment had a $0.66 \%$ effect in patients with type 2 diabetes, but the effect was not statistically significant. Although the literature included in this systematic review were interventional studies, some that did not randomize participants were also included $^{14)}$.

Therefore, this study aimed to assess the effects of periodontal treatment on $\mathrm{HbAlc}$ level in patients with type 2 diabetes through a systematic review on randomized controlled trial studies.

\section{Materials and Methods}

\section{Research design and participants}

This study is a systematic review of randomized controlled trials (RCTs) that aimed to answer the core question of "Does periodontal treatment effectively lower HbA1c levels in patients with type 2 diabetes?" Periodontal treatment was defined as SRP, and the main outcome variable was changes in the HbA1c levels.

\section{Inclusion and exclusion criteria}

1) RCTs, 2) patients in the experimental group who underwent nonsurgical SRP and patients in the control group who did not undergo periodontal treatment or received simple oral health education, 3) patients diagnosed with type 2 diabetes and periodontal disease, and 4) patients aged 18 years or older were included in the study.

1) Studies that included patients with systemic disease aside from type 2 diabetes, 2) patients who took medications known to affect periodontal tissue and treatment or those who received periodontal treatment in the last 3 months, and 3) patients who took antibiotics in the last 2 months were excluded from the study.

\section{Search strategy}

Search was conducted on April 8, 2018. All literatures that have been published up to date were searched without restricting the year of publication. MEDLINE, Embase, CINAHL, and the Cochrane Library were searched. The OpenGrey (www.opengrey.eu) and domestic periodontal medicine journals were searched to find gray literatures. For search keywords, combinations of "periodontal diseases" [MeSH], "dental prophylaxis" [MeSH], "dental scaling" $[\mathrm{MeSH}]$, "root planing" $[\mathrm{MeSH}]$, and "periodontal debridement" [MeSH] joined with "diabetes mellitus" [MeSH] and "diabetes mellitus, type 2" [MeSH] by "and" were used on PubMed. To increase search sensitivity, entry terms and "periodontal non-surgical treatment OR periodontal nonsurgical therapy OR scaling root planing OR periodontal treatment OR periodontal therapy OR calculus remov*" were additionally used in the search. A similar search method was used on other websites. 


\section{Literature selection}

Two researchers independently selected literatures according to the inclusion and exclusion criteria. During the first screening, literatures were selected based on their titles and abstracts. The second screening reviewed the full text of literatures. The two researchers selected the literatures to be included in the final analysis together.

\section{Statistical analysis}

To review the quality of the selected literatures, the data source, study design, target participants, included factors, interventions, and results were extracted. For quantitative integration of the data, the Review Manager (RevMan) statistical analysis program (ver. 5.3; The Nordic Cochrane Centre, The Cochrane Collaboration, Copenhagen, Denmark) was used. Q statistics and Higgin's $\mathrm{I}^{2}$ statistic were used to assess the heterogeneity degree of the selected literatures, and the inverse variance estimation method was used depending on the heterogeneity results to perform the meta-analysis.

\section{Results}

\section{Search results}

Fig. 1 shows the flowchart of literature search. Using the search strategy, a total of 571 were selected from MEDLINE, 440 literatures from the Cochrane Library,
Embase, and other search engines. Of the 1,011 literatures, 103 duplicates were removed. Next, 877 were removed based on their titles and abstracts. Of the remaining 31, 20 were selected following the full text review. These literatures included nine literatures that were not $\mathrm{RCTs}^{15-23)}$, six in which the method of periodontal treatment was not $\mathrm{SRP}^{13,24-28)}$, two in which the participants were not patients with type 2 diabetes and periodontal disease ${ }^{29,30)}$, one in which the outcome variable was not $\mathrm{HbA} 1 \mathrm{c}^{31)}$, and two without data ${ }^{32,33)}$.

\section{Meta-analysis for result estimation}

Table 1 summarizes the source, study design, participants, included factors, interventions, and results of the finally selected literatures ${ }^{4,34-43)}$.

In a study by Kiran et al. $^{34)}$, the experimental group showed significant decrease in dental plaque index, decrease gingival index, reduction in periodontal pocket depth, clinical adhesion loss, and bleeding gum $(\mathrm{p}<$ 0.001 ). The experimental group showed a significant decreased in HbAlc levels $(p<0.001)$, while the control group showed a slight increase in HbAlc levels ${ }^{34)}$.

Singh et al. ${ }^{35}$ reported that significant changes in periodontal indices were observed in experimental groups $\mathrm{A}$ and $\mathrm{B}$ following the periodontal treatment $(\mathrm{p}<0.001)$. A significant decrease in the HbA1c level relative to the level in the control groupwas observed in the experimental

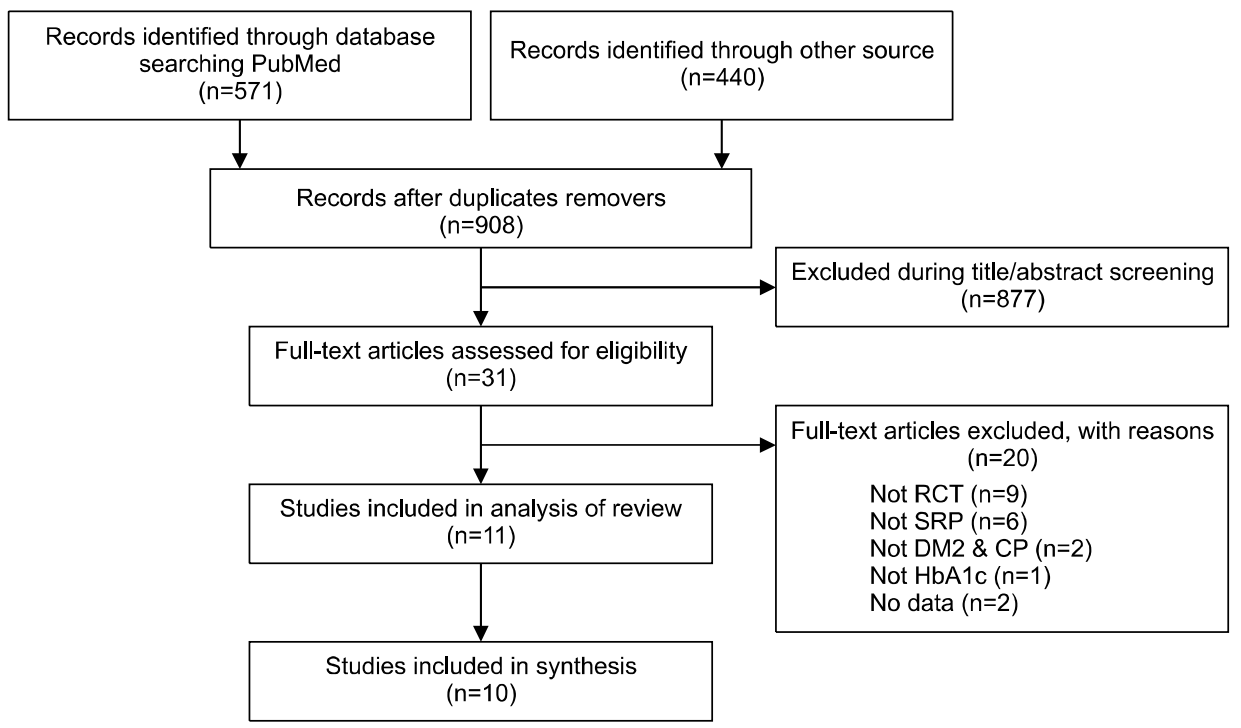

Fig. 1. Results of the search. RCT: randomized controlled trial, SRP: scaling and root planing, DM2: type 2 diabetes mellitus, CP: chronic periodontitis, HbA1c: glycated hemoglobin A. 


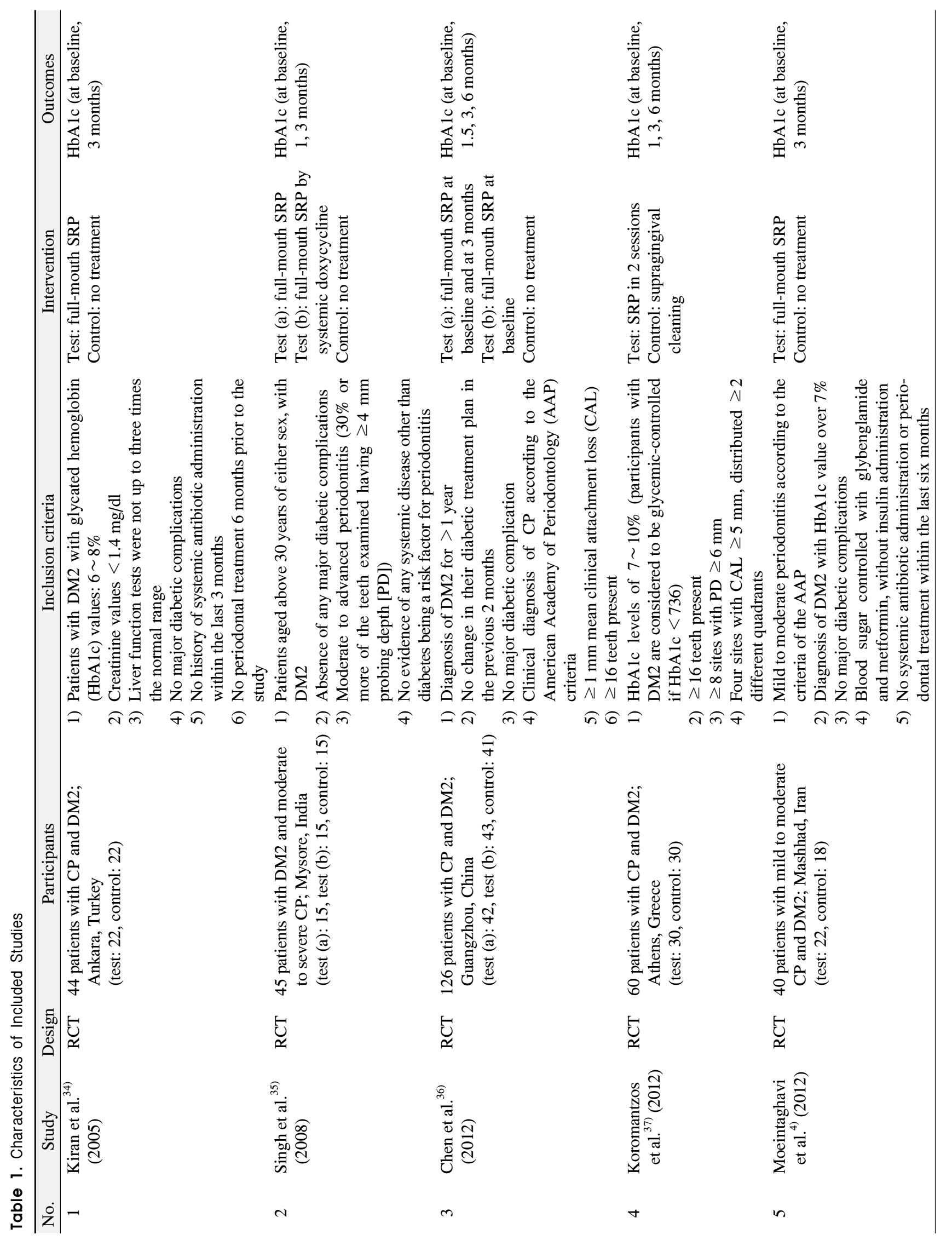




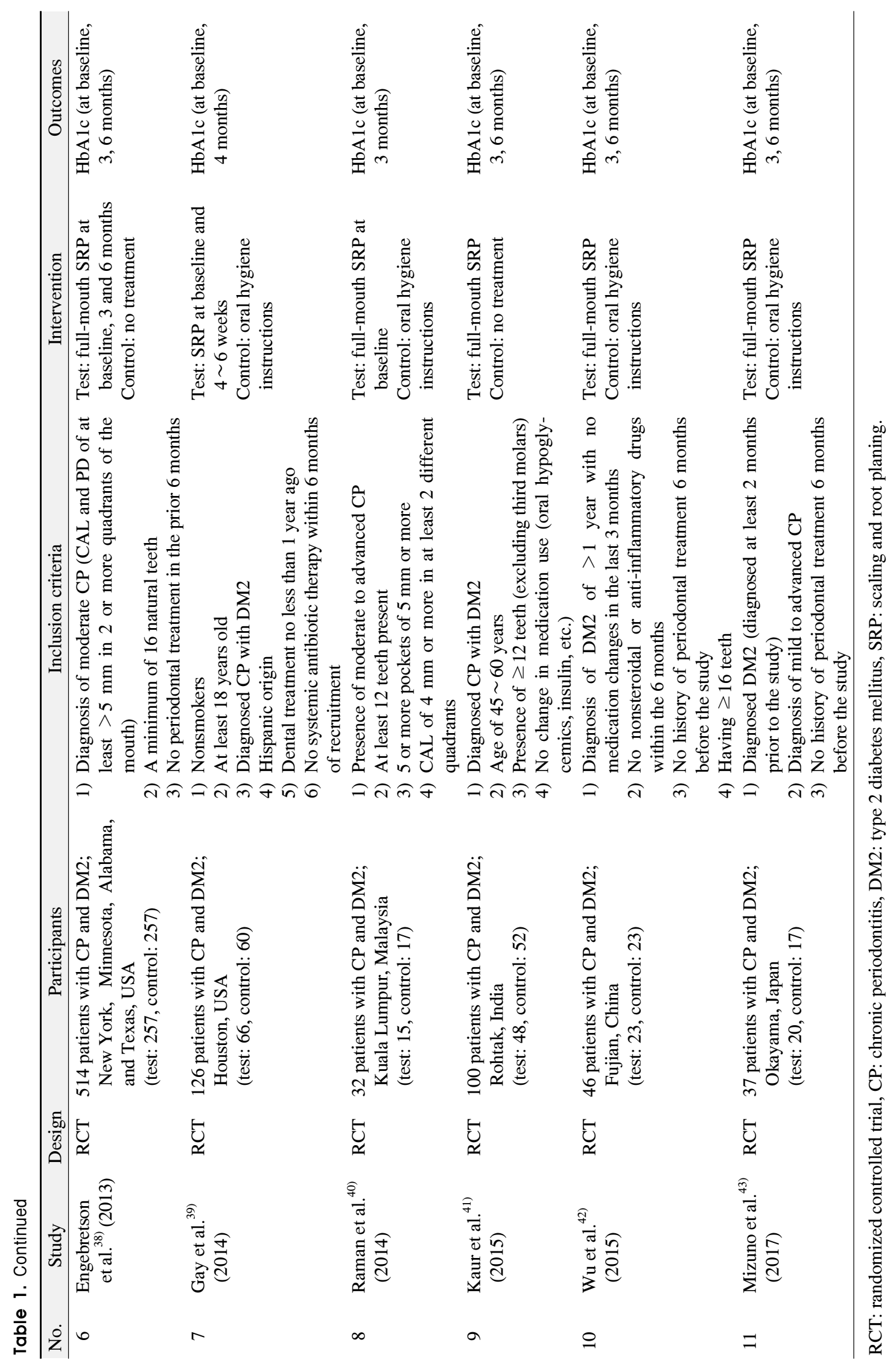


groups $(\mathrm{p}<0.001)^{35)}$.

Chen et al. ${ }^{36)}$ reported that significant decreases in the CRP level was observed in the two experimental groups following the periodontal treatment $(p<0.05)$. While the HbA1c level was significantly reduced in the second experimental group, no significant difference was observed in the HbA1c, fasting glucose, TNF- $\alpha$, and lipid levels between the two experimental groups $(\mathrm{p}>0.05)^{36}$.

Koromantzos et al. ${ }^{37)}$ reported that a significant decrease in the HbA1c level was observed following the nonsurgical periodontal treatment in patients with type 2 diabetes and severe periodontal disease $(\mathrm{p}<0.01)$. However, no significant difference was observed in the levels of hypersensitive CRP and matrix metalloproteinases 2 and $9^{37}$.

In a study by Moeintaghavi et al. ${ }^{4}$, a significant difference in the fasting glucose, HbA1c, total cholesterol, and cholesterol levels were observed at 3 months between the experimental and control groups $(\mathrm{p}=0.006, \mathrm{p}=0.003$, $\mathrm{p}<0.001$, respectively). At 3 months, the HbA1c level was significantly reduced in the experimental group ( $p=0.003$ ), while no significant change in the HbA1c level was observed in the control group ${ }^{4}$. Nonsurgical periodontal treatment was found to improve metabolic control in patients with diabetes ${ }^{4)}$.

Gay et al. $^{39)}$ showed that significant changes were observed in the clinical markers of oral health between the experimental and control groups at 4 months. However, no significant difference was observed in the changes of the
HbA1c level ${ }^{39)}$.

Raman et al. ${ }^{40)}$ showed that the HbA1c level significantly decreased in the experimental group at 3 months relative to the level during the early research period $(p=0.038)$. Although the HbA1c level in the control group also decreased at 3 months, the decrease was significantly insignificant. No significant difference was observed in the HbA1c level between the two groups at 3 months ${ }^{40}$.

Kaur et al. $^{41)}$ reported that the HbA1c level in the experimental group was significantly reduced at 3 and 6 months, and the decrease was significant when compared with the control group $(\mathrm{p}<0.05)$.

$\mathrm{Wu}$ et al. $^{42)}$ reported that no significant decrease was observed in the HbA1c level in the experimental group relative to the control group at 3 months. However, a significant decrease was observed at 6 months $(p=0.00)^{42}$.

In a study by Mizuno et al. ${ }^{43)}$, the balance of tissue oxidant capacity and the quality of life index significantly increased in the experimental group compared with the control group at 3 months. However, no significant difference was observed in the HbA1c level between the two groups ${ }^{43)}$.

The results of the meta-analysis demonstrated that SNP significantly reduced the $\mathrm{HbA} 1 \mathrm{c}$ level in patients with type 2 diabetes and periodontal disease (mean difference, $-0.34 ; 95 \%$ confidence interval, -0.43 to $-0.26 ; \mathrm{p}<$ 0.001) (Fig. 2).

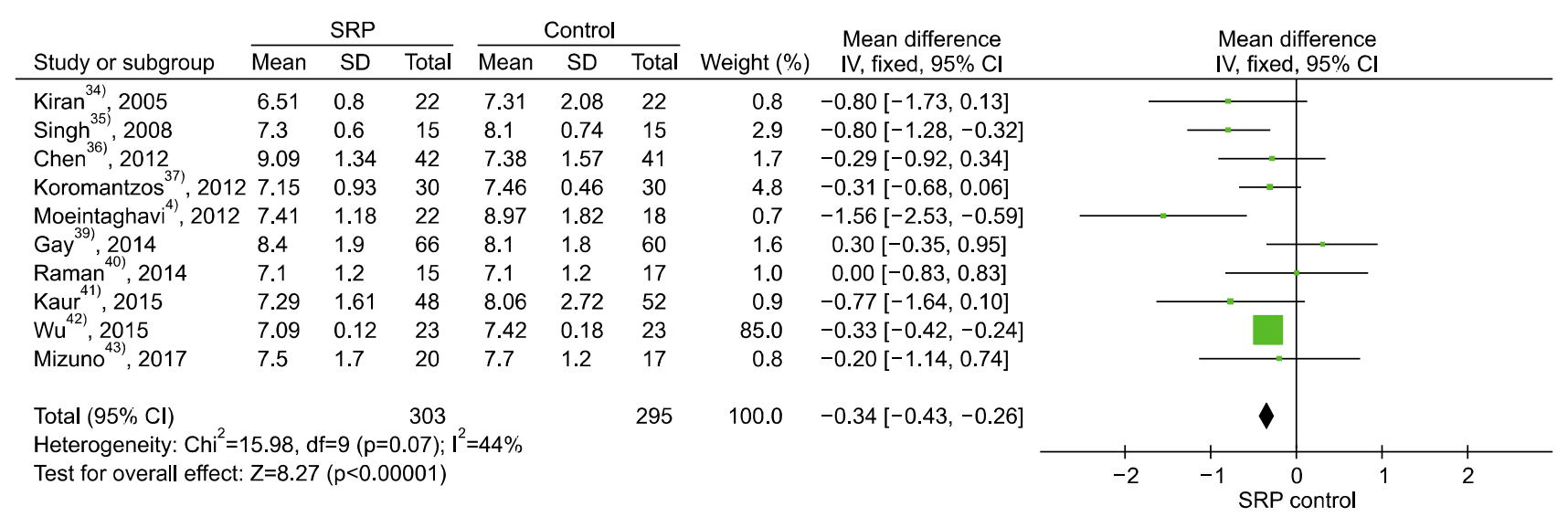

Fig. 2. Scaling and root planning (SRP) vs. no treatment. Glycated hemoglobin A difference between baseline and end of treatment. SD: standard deviation, IV: inverse variance estimation method, Cl: confidence interval, Chi ${ }^{2}: \mathrm{Q}$ statistic, df: degree of freedom, $\mathrm{I}^{2}$ : Higgin's $I^{2}$ statistic, Z: z-test. 


\section{Discussion}

This meta-analysis systematically reviewed RCTs to investigate the effects of periodontal treatment on the HbA1c level in patients with type 2 diabetes and periodontal disease. The results showed that SNP reduced the HbA1c level by 0.34 in patients with type 2 diabetes and periodontal disease, which is higher than the HbA1c reduction of 0.27 observed in a previous systematic review on the effects of nonsurgical periodontal treatment on HbA1c levels published in $2015^{44)}$.

In the previous study, nine studies were divided into two groups based on whether the number of participants was less than 80 , or greater than or equal to 80 , and were separately analyzed. The HbA1c level in the studies with less than 80 participants was more greatly reduced $(0.46)$ than that in the present study. The HbA1c level was slightly reduced (0.27) in the studies with 80 participants or more compared with that the present study.

Following the literature search and selection processes, seven literatures included in the previous meta-analysis and three studies that have been published after this meta-analysis were included in the final quantitative integration. Although the treatment method used for one of the two experimental groups in one of the nine literatures in the previous study corresponds to the treatment method used in the present study, the resulting values of the two experimental groups were combined in the analysis conducted in the previous study, making it difficult to assess the effects of SNP only. Hence, the results of the previous meta-analysis have limited interpretation $^{33)}$. The study by Engebretson et al. ${ }^{38)}$ included in the previous meta-analysis combined the results from various countries, and the standard deviation for the resulting values were not clearly provided. Therefore, the study was excluded from the final analysis of the present study. Since this study more strictly applied the standard intervention in the experimental group, it could accurately measure the effects of SNP on the HbA1c level. This study is also meaningful as it included literatures published until April 8, 2018, whereas the most recently published metaanalysis only searched literatures published until 2014.

Although studies on the association between periodontal disease and systemic disease have been actively conducted, studies on the effects of periodontal treatment on the HbA1c level in patients with type 2 diabetes and periodontal disease in Korea are limited, and there are almost no RCTs investigating the same. As the prevalence of diabetes increases due to the rapid aging of Korean population, it is necessary to conduct further research to evaluate the ability of periodontal treatment to improve the HbA1c level. Therefore clinical trials on Korean patients with diabetes are required considering the fact that the patients with type 2 diabetes are relatively old, the possibility of complex chronic diseases cannot be excluded due to the increasing number of patients with average age, and the high dropout rate of patients with diabetes.

In a study that analyzed the Fourth National Health and Nutrition Survey (2009), the risk of impaired fasting glucose increased as the severity of periodontal disease increased (odds ratio, 2.18; 95\% confidence interval, 1.92 $\sim 2.48 ; \mathrm{p}<0.001)^{10)}$. A study has been conducted on the association between periodontal and systemic diseases, and especially on the association between periodontal disease and diabetes. Improvements of systemic diseases through periodontal treatment based on the results of this study may be expected.

A study by Kiran et al. ${ }^{34)}$ included 44 patients; Singh et al. $^{35)}$, 45 patients; Moeintaghavi et al. ${ }^{4)}, 40$ patients; Raman et al. ${ }^{40)}$, 32 patients; Wu et al. ${ }^{42)}, 46$ patients; and that by Mizuno et al. ${ }^{43)}, 37$ patients. Although these sample sizes were statistically calculated, they are close to the minimum number of patients required, and these studies note the small sample sizes as their limitation. A study by Chen et al. ${ }^{36)}$ included 126 patients; Gay et al. ${ }^{39)}$, 126 patients; Kaur et al. ${ }^{41)}$, 100 patients. Since these studies included a larger number of patients compared with the aforementioned studies, they must have had a larger impact on the results of this meta-analysis.

The total length of the clinical trial period was 6 months in studies by Chen et al. ${ }^{36)}$, Koromantzos et al. ${ }^{37)}$, Kaur et al. $^{41)}$, and Wu et al. ${ }^{42)} ; 4$ months in a study by Gay et al. ${ }^{39)}$; and 3 months in the remaining studies. Since type 2 diabetes is a chronic disease that requires long-term care, regular periodontal treatment and long-term monitoring must be provided in patients with type 2 diabetes. Future 
meta-analysis on clinical trials must include long-term studies.

Besides the 10 literatures included in the final analysis, two literatures satisfied the inclusion criteria. However, they were not included in the final analysis because their result values were not clear in the data extraction process. A study by Engebretson et al. ${ }^{38)}$ had a relatively larger sample size of 514 patients compared with other studies, but the results had missing standard deviations. Thus, all studies could not be included in the analysis because the results remained unclear, and this was considered a limitation of this study.

Despite this, this study is considered significant as it demonstrates that periodontal treatment can improve the HbA1c level and provides a basis for the management of diabetes complications, whose incidence has been increasing.

Various studies are being conducted to evaluate the association between periodontal and systemic diseases. The need for management using nonsurgical periodontal treatments, such as SRP, to improve the quality of life and reduce the burden of medical costs is especially emphasized for diabetes.

\section{References}

1. Shaw JE, Sicree RA, Zimmet PZ: Global estimates of the prevalence of diabetes for 2010 and 2030. Diabetes Res Clin Pract 87: 4-14, 2010. https://doi.org/10.1016/j.diabres.2009.10.007

2. Jo EK, Seo EW, Lee KS: Spatial distribution of diabetes prevalence rates and its relationship with the regional characteristics. Korean Soc Health Policy Adm 26: 30-38, 2016. https://doi.org/10.4332/KJHPA.2016.26.1.30

3. Green A, Christian Hirsch N, Pramming SK: The changing world demography of type 2 diabetes. Diabetes Metab Res Rev 19: 3-7, 2003. https://doi.org/10.1002/dmrr.340

4. Moeintaghavi A, Arab HR, Bozorgnia Y, Kianoush K, Alizadeh M: Non-surgical periodontal therapy affects metabolic control in diabetics: a randomized controlled clinical trial. Aust Dent J 57: 31-37, 2012. https://doi.org/10.1111/j.1834-7819.2011.01652.x

5. Bascones-Martínez A, González-Febles J, Sanz-Esporrín J:
Diabetes and periodontal disease. Review of the literature. Am J Dent 27: 63-67, 2014.

6. Mealey BL, Oates TW; American Academy of Periodontology: Diabetes mellitus and periodontal diseases. J Periodontol 77: 1289-1303, 2006.

https://doi.org/10.1902/jop.2006.050459

7. Taylor GW, Burt BA, Becker MP, et al.: Severe periodontitis and risk for poor glycemic control in patients with noninsulin-dependent diabetes mellitus. J Periodontol 67 Suppl 10S: 1085-1093, 1996. https://doi.org/10.1902/jop.1996.67.10s.1085

8. Han SB: Practice of non surgical periodontal treatment. J Korean Dent Assoc 46: 352-361, 2008.

9. Jung JO, Lee KH, Youn HJ: The correlation between diabetes and periodontitis in Korean adults. J Dent Hyg Sci 11: 221-228, 2011.

10. Won $\mathrm{JH}, \mathrm{Ha} \mathrm{MN}$ : An association of periodontitis and diabetes. J Dent Hyg Sci 14: 107-113, 2014.

11. Kim SY, Jang HG: Influence of metabolic on periodontal disease in Korean adults. J Korean Soc Dent Hyg 15: 399-410, 2015 https://doi.org/10.13065/jksdh.2015.15.03.399

12. Iwamoto $Y$, Nishimura F, Nakagawa $M$, et al.: The effect of antimicrobial periodontal treatment on circulating tumor necrosis factor-alpha and glycated hemoglobin level in patients with type 2 diabetes. J Periodontol 72: 774-778, 2001. https://doi.org/10.1902/jop.2001.72.6.774

13. Jones JA, Miller DR, Wehler CJ, et al.: Does periodontal care improve glycemic control? The Department of Veterans Affairs Dental Diabetes Study. J Clin Periodontol 34: 46-52, 2007. https://doi.org/10.1111/j.1600-051X.2006.01002.x

14. Janket SJ, Wightman A, Baird AE, Van Dyke TE, Jones JA: Does periodontal treatment improve glycemic control in diabetic patients? A meta-analysis of intervention studies. J Dent Res 84: 1154-1159, 2005. https://doi.org/10.1177/154405910508401212

15. Simpson TC, Needleman I, Wild SH, Moles DR, Mills EJ: Treatment of periodontal disease for glycaemic control in people with diabetes. Cochrane Database Syst Rev (5): CD004714, 2010. https://doi.org/10.1002/14651858.CD004714.pub2

16. Steffens JP, Glaci Reinke SM, Angel Muñoz M, Santos FA, Luiz Pilatti G: Review on periodontal disease and metabolic 
control of diabetes mellitus. Rev Med Chil 138: 1172-1178, 2010. https://doi.org/10.4067/S0034-98872010000900014

17. Calabrese N, D'Aiuto F, Calabrese A, Patel K, Calabrese G, Massi-Benedetti M: Effects of periodontal therapy on glucose management in people with diabetes mellitus. Diabetes Metab 37: 456-459, 2011.

https://doi.org/10.1016/j.diabet.2011.05.004

18. Santos VR, Ribeiro FV, Lima JA, et al.: Partial- and full-mouth scaling and root planing in type 2 diabetic subjects: a 12-mo follow-up of clinical parameters and levels of cytokines and osteoclastogenesis-related factors. J Periodontal Res 47: 45-54, 2012. https://doi.org/10.1111/j.1600-0765.2011.01403.x

19. Obradović R, Kesić L, Mihailović D, et al.: A histological evaluation of a low-level laser therapy as an adjunct to periodontal therapy in patients with diabetes mellitus. Lasers Med Sci 28: 19-24, 2013. https://doi.org/10.1007/s10103-012-1058-7

20. Casanova L, Hughes FJ, Preshaw PM: Diabetes and periodontal disease: a two-way relationship. Br Dent J 217: 433-437, 2014. https://doi.org/10.1038/sj.bdj.2014.907

21. Mauri-Obradors E, Jané-Salas E, Sabater-Recolons Mdel M, Vinas M, López-López J: Effect of nonsurgical periodontal treatment on glycosylated hemoglobin in diabetic patients: a systematic review. Odontology 103: 301-313, 2015. https://doi.org/10.1007/s10266-014-0165-2

22. Simpson TC, Weldon JC, Worthington HV, et al.: Treatment of periodontal disease for glycaemic control in people with diabetes mellitus. Cochrane Database Syst Rev (11): CD004714, 2015. https://doi.org/10.1002/14651858.CD004714.pub3

23. Botero JE, Rodríguez C, Agudelo-Suarez AA: Periodontal treatment and glycaemic control in patients with diabetes and periodontitis: an umbrella review. Aust Dent J 61: 134-148, 2016. https://doi.org/10.1111/adj.12413

24. Deo V, Gupta S, Bhongade ML, Jaiswal R: Evaluation of subantimicrobial dose doxycycline as an adjunct to scaling and root planing in chronic periodontitis patients with diabetes: a randomized, placebo-controlled clinical trial. J Contemp Dent Pract 11: 009-016, 2010.

25. Lin SJ, Tu YK, Tsai SC, Lai SM, Lu HK: Non-surgical periodontal therapy with and without subgingival minocycline administration in patients with poorly controlled type II diabetes: a randomized controlled clinical trial. Clin Oral Investig 16: 599-609, 2012.

https://doi.org/10.1007/s00784-011-0535-x

26. Nassar PO, Walker CS, Salvador CS, Felipetti FA, Orrico SR, Nassar CA: Lipid profile of people with diabetes mellitus type 2 and periodontal disease. Diabetes Res Clin Pract 96: 35-39, 2012. https://doi.org/10.1016/j.diabres.2011.11.017

27. Pradeep AR, Sharma A, Rao NS, Bajaj P, Naik SB, Kumari M: Local drug delivery of alendronate gel for the treatment of patients with chronic periodontitis with diabetes mellitus: a double-masked controlled clinical trial. J Periodontol 83: 1322-1328, 2012. https://doi.org/10.1902/jop.2012.110292

28. Gelato MC, Schoenfeld E, Hou W, et al.: Changes in diabetes medications in the Diabetes and Periodontal Therapy Trial and their effect on hemoglobin A1c (HbA1c). Contemp Clin Trials 50: 21-27, 2016. https://doi.org/10.1016/j.cct.2016.07.010

29. Engebretson SP, Hey-Hadavi J: Sub-antimicrobial doxycycline for periodontitis reduces hemoglobin A1c in subjects with type 2 diabetes: a pilot study. Pharmacol Res 64: 624-629, 2011. https://doi.org/10.1016/j.phrs.2011.06.024

30. Mendonça AC, Santos VR, Ribeiro FV, et al.: Surgical and non-surgical therapy with systemic antimicrobials for residual pockets in type 2 diabetics with chronic periodontitis: a pilot study. J Clin Periodontol 39: 368-376, 2012. https://doi.org/10.1111/j.1600-051X.2012.01860.x

31. Michalowicz BS, Hyman L, Hou W, et al.: Factors associated with the clinical response to nonsurgical periodontal therapy in people with type 2 diabetes mellitus. J Am Dent Assoc 145: 1227-1239, 2014. https://doi.org/10.14219/jada.2014.92

32. Vergnes JN, Arrivé E, Gourdy P, et al.: Periodontal treatment to improve glycaemic control in diabetic patients: study protocol of the randomized, controlled DIAPERIO trial. Trials 10: 65, 2009. https://doi.org/10.1186/1745-6215-10-65

33. Zhang H, Li C, Shang S, Luo Z: Scaling and root planing with enhanced root planing on healthcare for type 2 diabetes mellitus: a randomized controlled clinical trial. J Dent Sci 8: 272-280, 2013. https://doi.org/10.1016/j.jds.2012.10.009

34. Kiran M, Arpak N, Unsal E, Erdogan MF: The effect of improved periodontal health on metabolic control in type 2 diabetes mellitus. J Clin Periodontol 32: 266-272, 2005. https://doi.org/10.1111/j.1600-051X.2005.00658.x

35. Singh S, Kumar V, Kumar S, Subbappa A: The effect of 
periodontal therapy on the improvement of glycemic control in patients with type 2 diabetes mellitus: a randomized controlled clinical trial. Int J Diabetes Dev Ctries 28: 38-44, 2008.

36. Chen L, Luo G, Xuan D, et al.: Effects of non-surgical periodontal treatment on clinical response, serum inflammatory parameters, and metabolic control in patients with type 2 diabetes: a randomized study. J Periodontol 83: 435-443, 2012. https://doi.org/10.1902/jop.2011.110327

37. Koromantzos PA, Makrilakis K, Dereka X, et al.: Effect of non-surgical periodontal therapy on C-reactive protein, oxidative stress, and matrix metalloproteinase (MMP)-9 and MMP-2 levels in patients with type 2 diabetes: a randomized controlled study. J Periodontol 83: 3-10, 2012. https://doi.org/10.1902/jop.2011.110148

38. Engebretson SP, Hyman LG, Michalowicz BS, et al.: The effect of nonsurgical periodontal therapy on hemoglobin A1c levels in persons with type 2 diabetes and chronic periodontitis: a randomized clinical trial. JAMA 310: 2523-2532, 2013. https://doi.org/10.1001/jama.2013.282431

39. Gay IC, Tran DT, Cavender AC, et al.: The effect of periodontal therapy on glycaemic control in a Hispanic population with type 2 diabetes: a randomized controlled trial. J Clin Periodontol 41: 673-680, 2014.

https://doi.org/10.1111/jcpe.12268

40. Raman RP, Taiyeb-Ali TB, Chan SP, Chinna K, Vaithilingam
RD: Effect of nonsurgical periodontal therapy verses oral hygiene instructions on type 2 diabetes subjects with chronic periodontitis: a randomised clinical trial. BMC Oral Health 14: 79, 2014. https://doi.org/10.1186/1472-6831-14-79

41. Kaur PK, Narula SC, Rajput R, Sharma KR, Tewari S: Periodontal and glycemic effects of nonsurgical periodontal therapy in patients with type 2 diabetes stratified by baseline HbA1c. J Oral Sci 57: 201-211, 2015. https://doi.org/10.2334/josnusd.57.201

42. Wu Y, Chen L, Wei B, Luo K, Yan F: Effect of non-surgical periodontal treatment on visfatin concentrations in serum and gingival crevicular fluid of patients with chronic periodontitis and type 2 diabetes mellitus. J Periodontol 86: 795-800, 2015. https://doi.org/10.1902/jop.2015.140476

43. Mizuno H, Ekuni D, Maruyama T, et al.: The effects of non-surgical periodontal treatment on glycemic control, oxidative stress balance and quality of life in patients with type 2 diabetes: a randomized clinical trial. PLoS One 12: e0188171, 2017. https://doi.org/10.1371/journal.pone.0188171

44. Li Q, Hao S, Fang J, Xie J, Kong XH, Yang JX: Effect of non-surgical periodontal treatment on glycemic control of patients with diabetes: a meta-analysis of randomized controlled trials. Trials 16: 291, 2015.

https://doi.org/10.1186/s13063-015-0810-2 